\title{
Oral Session 7: Suborbital and Parabolic Flight
}

\section{7-1 [\#3033]}

\section{Tactile cueing as a gravitational substitute for spatial navigation during parabolic flight}

K.L. Montgomery ${ }^{\mathrm{a}}$, K.H. Beaton ${ }^{\mathrm{b}}$, J.M. Barbac ${ }^{\mathrm{c}}$, J.M. Cackler $^{\text {a }}$, J.H. Son ${ }^{\text {a }}$, S.P. Horsfield ${ }^{\text {d }}$ and S.J. Wood ${ }^{\mathrm{e}}$

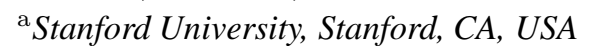

b Johns Hopkins University, Baltimore, MD, USA

${ }^{\mathrm{c}}$ Texas A\&M University, College Station, TX, USA

d Ohio University, Athens, OH, USA

${ }^{\mathrm{e}}$ NASA Johnson Space Center and Universities Space Research Association, Houston, TX, USA

Introduction: Spatial navigation requires an accurate awareness of orientation in your environment. The purpose of this experiment was to examine how spatial awareness was impaired with changing gravitational cues during parabolic flight, and the extent to which vibrotactile feedback of orientation could be used to help improve performance.

Methods: Six subjects were restrained in a chair tilted relative to the plane floor, and placed at random positions during the start of the microgravity phase. Subjects reported their orientation using verbal reports, and used a hand-held controller to point to a desired target location presented using a virtual reality video mask. This task was repeated with and without constant tactile cueing of "down" direction using a belt of 8 tactors placed around the mid-torso. Control measures were obtained during ground testing using both upright and tilted conditions.

Results: Perceptual estimates of orientation and pointing accuracy were impaired during microgravity or during rotation about an upright axis in $1 \mathrm{~g}$. The amount of error was proportional to the amount of chair displacement. Perceptual errors were reduced during movement about a tilted axis on earth.
Conclusions: Reduced perceptual errors during tilts in $1 \mathrm{~g}$ indicate the importance of otolith and somatosensory cues for maintaining spatial awareness. Tactile cueing may improve navigation in operational environments or clinical populations, providing a non- visual non-auditory feedback of orientation or desired direction heading.

Acknowledgement: Support was provided by NSBRI through NASA NCC 9-58 (NA0405).

\section{7-2 [\#3034]}

\section{Gravity dependence of the pitch vestibulo-ocular reflex}

K.H. Beaton ${ }^{\mathrm{a}}$, F. Karmali ${ }^{\mathrm{b}}$ and M. Shelhamer ${ }^{\mathrm{a}}$

${ }^{a}$ Department of Biomedical Engineering, Johns Hopkins University School of Medicine, Baltimore, MD, USA

b Jenks Vestibular Physiology Laboratory, Massachusetts Eye and Ear Infirmary/Harvard Medical School, Boston, MA, USA

Head movements in the pitch plane are especially relevant to sensorimotor function following spaceflight, as many astronauts have reported hypersensitivity to pitching movements during re- adaptation to a $1 \mathrm{~g}$ environment. This study focuses on changes in the pitch vestibulo-ocular reflex (pVOR) in different gravity levels. While the pVOR is primarily driven by the vertical semicircular canals, considerable evidence from clinical and spaceflight studies has demonstrated that it is modulated by otolith input. Here, we present the results of several parabolic flight studies in which we measured the pVOR, either directly via simultaneous measurements of head and eye movements, or via a new technique that we have entitled vestibulo-ocular nulling (VON). In VON, a rate sensor measures head 
movement and feeds it to a computer, which displays a visual target. As the head moves, the device measures head motion and uses it to control target position. The subject controls the gain of the target motion (relative to head motion) in order to reduce the apparent motion of the target to zero. Because the motion-gain value set by the subject is inversely proportional to the desired VOR gain, the motion- gain value set by the subject provides a surrogate measure of vestibulo-ocular function. In general, during parabolic flights, subjects showed differences in VOR gain in different g-levels, as predicted based on otolith stimulation. These differences decreased with experience, so that gains were similar in the various g-levels after adaptation, suggesting a context-specific otolith contribution.

\section{7-3 [\#3030]}

\section{An agenda for sensorimotor research in sub-orbital flight}

F. Karmali ${ }^{\mathrm{a}}$ and M. Shelhamer ${ }^{\mathrm{b}}$

${ }^{a}$ Jenks Vestibular Physiology Laboratory, Massachusetts Eye and Ear Infirmary/Harvard Medical School, Boston, MA, USA

${ }^{\mathrm{b}}$ Department of Biomedical Engineering, Johns Hopkins University School of Medicine, Baltimore, MD, USA

The excitement of space travel will be open to thousands of people by new commercial sub-orbital operations. Experience with changing g levels during space flight and parabolic flight suggests that sensorimotor disruptions are likely in these travelers, including postural stability, eye movements and motor coordination. We believe overcoming these sensorimotor disruptions will require aframework that delineates how approaches should differ from those applied to orbital flight and between sub-orbital passengers and pilots. For example, while most passengers are interested in maximizing enjoyment and flying only once, pilots are interested in maximizing precision and safety, and fly often. Strategies for overcoming disruptions include sensorimotor adaptation, re-adaptation, pre-adaptation, pharmaceuticals and cognitive training.

Sensorimotor adaptation is one strategy for overcoming disruptions. However, its application to sub-orbital flight, with periods of reduced and enhanced gravity lasting less than four minutes, is an open question. We have performed experiments on sensorimotor adapta- tion during parabolic flight, in which we tested subjects over four consecutive days of flying. The reflex we tested, the pitch vestibulo-ocular reflex, took a few days during to overcome an initial disruption. This suggests that sensorimotor adaptation will be important for sub-orbital pilots, and that sub-orbital passengers may benefit from previous exposure to parabolic flight.

To improve comfort and safety during sub-orbital operations, we recommend using parabolic flight to preadapt sub-orbital passengers, and we recommend continued research to understand the best timing for these flights, and tasks for passengers to conduct. We recommend emphasizing recency of experience for suborbital pilots.

\section{7-4 [\#3061]}

\section{Pre-flight adaptation protocols for suborbital flight}

\author{
M. Shelhamer and K.H. Beaton \\ Department of Biomedical Engineering, Johns Hopkins \\ University School of Medicine, Baltimore, MD, USA
}

Commercial suborbital flights will present a challenging sensorimotor experience, with 4-5 minutes of $0 \mathrm{~g}$ between hyper-g launch and landing phases. Based on experience with parabolic flight, and the likely wide range of fitness and experience levels of suborbital passengers, sensorimotor disturbances are very likely to be a problem (motion sickness, disorientation, discomfort and anxiety). Pre-flight adaptation protocols might alleviate some of these issues. We are developing a set of sensorimotor assessment tests for use after longduration space flight. A subset of these tests can be applied to passengers before and after suborbital flight. The tests include rotational and translational vestibuloocular assessment, ocular skew and disconjugate torsion, and roll vection. Performance on these tests can be examined for correlations with in-flight experience (motion sickness, etc.) based on questionnaires and cabin video recordings. Through an understanding of adaptation to parabolic and orbital flight, obtained from many previous studies, we can then suggest appropriate pre-flight adaptation procedures. Available preflight adaptation protocols include parabolic flight and centrifugation, and related concepts include contextspecific adaptation and adaptive generalization. Coupling each of these to the appropriate passenger, based on pre-flight testing, is the challenge. As one example, if incorrect canal-otolith integration is identified, based 
on pitch and roll VOR testing, then pre-flight training of the pitch and roll VOR in parabolic flight might be of use. The premise is that the appropriate $0 \mathrm{~g}$-specific sensorimotor program would be learned ahead of time, and called into play once in $0 \mathrm{~g}$ suborbital flight. Such a research program would not only provide information on human adaptation to flight, but could also improve the flight experience for passengers, and involve them as part of the scientific enterprise rather than simply as thrill seekers. Testing of suborbital passengers should begin as soon as possible after flights begin, to quickly establish the required database to enable this work.

Acknowledgement: Supported by NASA NNX10A O19G. 